\title{
Terahertz Time-Domain Study of Silver Nanoparticles Synthesized by Laser Ablation in Organic Liquid
}

\author{
Can Koral, Bülend Ortaç, and Hakan Altan
}

\begin{abstract}
We report the investigation of laser-synthesized Ag nanoparticles (Ag-NPs) in an organic liquid environment by using terahertz time-domain spectroscopy (THz-TDS) technique. Colloidal Ag-NPs with an average diameter of $10 \mathrm{~nm}$ in twopropanol solution through nanosecond pulsed laser ablation were synthesized. THz-TDS measurements were performed on different volumetric concentration of Ag-NPs suspensions placed in 2-mm path length quartz cuvette. Due to the dispersive and highly absorptive nature of the nano liquids, an approach based on extracting the optical properties through the changes in amplitude and phase solely around the main peak of $\mathrm{THz}$ waveform is developed. This approach allowed for an accurate estimation of the complex refractive index of the Metallic-NPs suspension for the different prepared volumetric concentrations. In addition, using MaxwellGarnett theory, the NP concentration is also extracted. This method shows that the time-domain nature of the THz pulse measurement technique is extremely useful in instances where slight variations in highly dispersive samples need to be investigated.
\end{abstract}

Index Terms- THz applications in chemistry and spectroscopy, THz modeling and analysis techniques.

\section{INTRODUCTION}

$\mathbf{N}$ ANOTECHNOLOGY has a broad range of potential applications combining contributions from physics, chemistry, biology, materials science, engineering, and many other disciplines. The mechanical, magnetic, chemical, electronic, and optical properties of materials are size dependent so that those properties of nanostructured materials can be tailored controllably by modifying the size and shape or extent of agglomeration [1], [2]. Furthermore, the addition of nanomaterials to a variety of host media can enhance structural, electrical, and thermal properties [3], [4]. To that extent, construction of tools for characterization, detection of nanosized materials is of great importance. Terahertz time-domain spectroscopy (THz-TDS) has evolved into an excellent tool for the investigation into the properties of bulk or even nanometer-sized materials [5]. It is also important to note that $\mathrm{THz}$ radiation is safe for humans due to its low photon energy, and also is nondestructive to samples

Manuscript received August 31, 2015; revised January 19, 2016, April 03, 2016, April 19, 2016, and May 11, 2016; accepted May 19, 2016. Date of publication June 14, 2016; date of current version July 08, 2016.

C. Koral is with the Department of Chemistry, University of Bari, Bari 70126, Italy (e-mail: can.koral@nanotec.cnr.it).

B. Ortac is with the Institute of Materials Science and Nanotechnology, UNAM, Bilkent University, Ankara 06800, Turkey (e-mail: ortac@unam. bilkent.edu.tr).

H. Altan is with the Department of Physics, Middle East Technical University, Ankara 06800, Turkey (e-mail: haltan@metu.edu.tr).

Color versions of one or more of the figures in this paper are available online at http://ieeexplore.ieee.org.

Digital Object Identifier 10.1109/TTHZ.2016.2572360
[2], [5] and can be used safely for characterizing explosives [6,7] and flammable fluids [8], [9].

Even though the $\mathrm{THz}$ wavelength is much larger than the size of NPs, it can still be used to analyze a group of NPs since metals are near perfect reflectors in the far infrared [10]. One of the major advantages of THz-TDS is that the transient THz electric field is measured, and therefore, the constituent elements of the pulse which are the amplitude and phase that have connection with the absorption coefficient and the refractive index of the sample can be determined [10]-[12]. Another advantage is that in this range wavelengths are long enough not to be absorbed by nonpolar and nonmetallic materials. In this study, we developed a novel method for the characterization of the properties of metallic NPs based on terahertz radiation and its interaction with conducting media. The changes of the amplitude and phase of $\mathrm{THz}$ pulse interacting with the Ag-NPs in organic liquid environment allows for the extraction of the effective complex refractive index of the Ag-NPs suspension, and hence, the different concentrations of the NPs if one knows beforehand the refractive index of the host NP. To our knowledge, quantifying Metallic NPs with this new method is completely novel and has not been implemented before in the open scientific literature.

\section{METHODS}

The THz field strongly interacts with polar molecules and polar liquids tend to absorb the THz intensity; thus, when studying the properties of the materials in such liquids, researchers typically use very thin path lengths [13]. In order to better analyze nanofluids, it is important to determine the host fluid with minimum absorption coefficient in the $\mathrm{THz}$ frequency range. While the use of nonpolar liquids can be an advantage due to increased $\mathrm{THz}$ transmission, it can also be disadvantageous for NP suspensions, since the use of polar liquids tend to stop aggregation [14]. A home-built THz-TDS system was utilized for all the measurements. The THz-TDS system is driven by a sub- $20 \mathrm{fs} \mathrm{Ti}: \mathrm{Al}_{2} \mathrm{O}_{3}$ laser source (Coherent Verdi-V5 pumped Femtosource Femtolaser Scientific XLPro). The system uses a photoconductive antenna (Batop PCA-40-05-10-800) for THz generation and a 2-mm-thick $<110>\mathrm{ZnTe}$ crystal for $\mathrm{THz}$ detection based on the electro-optic method. Off-axis parabolic metal reflectors and TPX (polymethypenetene) lenses were used to focus and collimate the broad $\mathrm{THz}$ frequency range onto both the host liquids and NPs placed in a 2-mm path length quartz cuvette with $1 \mathrm{~cm}$ of width. In order to select the ideal polar host fluid, we performed measurements for pure Ethanol (99,5\%), Ethylene 
glycol (99\%), 2-propanol (99,5\%), Methanol (95\%), and Distilled water without the presence of Ag-NPs. We obtained the time-domain data sets with a step size of 20 microns which result in an error of $+/-0.0333$ ps per step. As a result, 2-propanol was found to have minimum absorption loss in the time domain compared to other possible hosts.

\section{AG-NPS SyNTHESIS By LASER ABLATION IN LIQUID}

Ag-NPs suspensions measured in these studies were manufactured using pulsed laser ablation method [15], [16] in 2propanol host media. Ag-NPs were synthesized by using pulsed Nd:YLF laser with $527 \mathrm{~nm}$ wavelength, $16 \mathrm{~W}$ average power, $110 \mathrm{~ns}$ pulse duration, and $16 \mathrm{~mJ}$ pulse energy for $1 \mathrm{kHz}$. The laser focused on the pure $\mathrm{Ag}$ (99.999\%, Kurt J. Lesker) target located in $10 \mathrm{ml}$ 2-propanol by a lens with focal length of 50 $\mathrm{mm}$. The ablation was carried out for $5 \mathrm{~min}$ for synthesis. Ablation of Ag-NPs immersed in 2-propanol results in a yellow hue. The morphology and the diameter of the Ag-NPs were investigated by transmission electron microscope (TEM) (FEI-Tecnai G2F30). The size and the structural characteristics of Ag-NPs demonstrate that the colloidal Ag-NPs is synthesized with spherical shape within 10-nm average particle sizes as was deduced from TEM images [see Fig. 1(a)]. The particle size distribution is furthermore confirmed by Zeta Potential Analyze Method [inset Fig. 1(b)] [17] [predominant average particle of size of around $10-15 \mathrm{~nm}(80 \%)$ with second and third particle sizes with an occurrence of $15-20 \mathrm{~nm}(15 \%)$, and 5-10 nm (5\%)]. The elemental compositions of the colloidal Ag-NPs [inset Fig. 1(a)] were obtained by EDAX analysis. The presence of the Ag peak in the EDAX spectrum confirms that Ag nanoparticles were successfully generated by laser ablation technique. Fig. 1(b) presents the HRTEM image of a $10 \mathrm{~nm}$ Ag-NP. HRTEM image of a single isolated $\mathrm{Ag}-\mathrm{NP}$ shows the crystalline lattice fringes which confirm crystalline Ag-NPs generation by nanosecond pulsed laser ablation technique in liquid environment.

\section{THZ-TDS NP SUSPENSION MEASUREMENTS}

Our preliminary analysis showed that both the concentration of NPs and refractive index of the metal particles are important to observe an appreciable change in the delay in the time-domain pulse in the $\mathrm{THz}$ transmission. Due to the nonaggregate nature of the suspension, the nanoparticles were well separated. This allowed to treat each one individually in its interaction with the $\mathrm{THz}$ field. Noting that the dimension of the nanoparticle has little effect on the real part of the dielectric function, the refractive index of the bulk material obtained from reflectance measurements was used to estimate the refractive index of the nanoparticles [18], [19].

The predominant size of the NPs was found to be close to $10 \mathrm{~nm}$. This estimation of the size of NPs affects the overall particle concentration that is to be extracted since the fill factor for spherical particles in a medium is proportional to the cube of the particle radius. For this reason, an accurate estimation of the particle radius is necessary for accurate determination of the particle concentration. We expected the THz pulse to experience both amplitude and phase change upon passing through the NPs

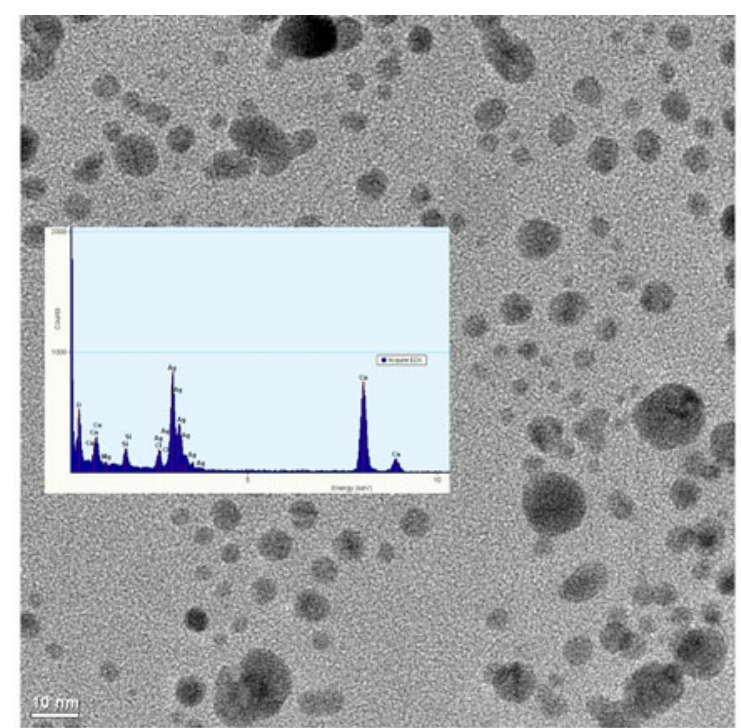

(a)

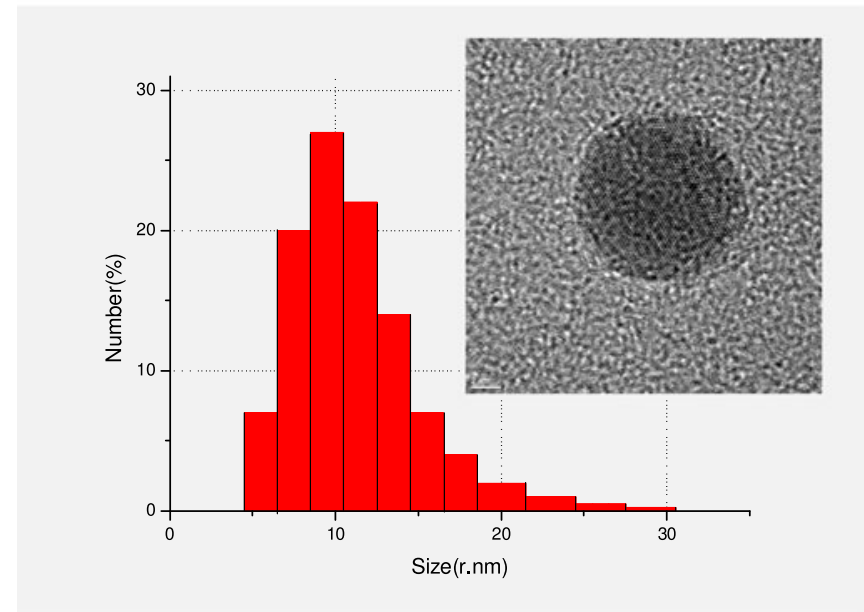

(b)

Fig. 1. (a) TEM image of NPs and the EDX profile shown. (b) HRTEM image of a single Ag-NP, and the Zeta Potential data.

suspensions. For this reason, measurements were performed for 2-proponol solutions with and without the presence of Ag-NPs under different concentrations.

Different concentrations of NPs suspensions (where three quarters, one-half and one quarter concentrations by volume were prepared and labeled based on the original sample of nonaggregating Ag NP in 2-propanol liquid) were prepared by direct diluting with (99.5\%) 2-propanol. The Ag- NPs ratio to the host 2-propanol by volume in those samples was as follows: $0.75 \mathrm{Ag}-\mathrm{NPs}, 0.5 \mathrm{Ag}-\mathrm{NPs}$ and $0.25 \mathrm{Ag}-\mathrm{NPs}$.

The THz-TDS measurements were done by loading the liquids without removing the sample holder so as to ensure that the effects due to the placement of the sample holder were eliminated. Since the varying concentration of NPs suspensions did not have an appreciable shift in the time domain waveform, the measurements were taken with higher resolution than typically employed in THz-TDS measurements. The frequencydependent quantities such as power absorption coefficient and 


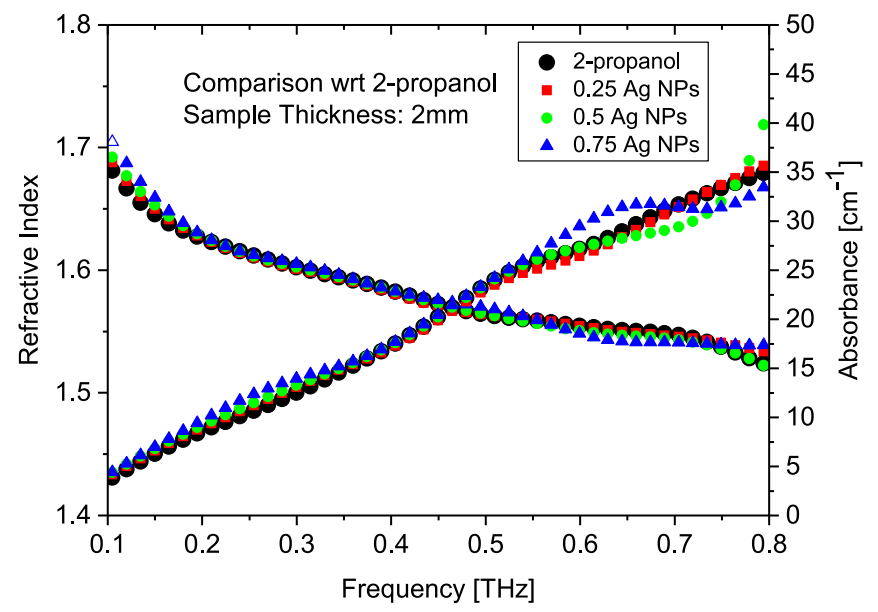

(a)

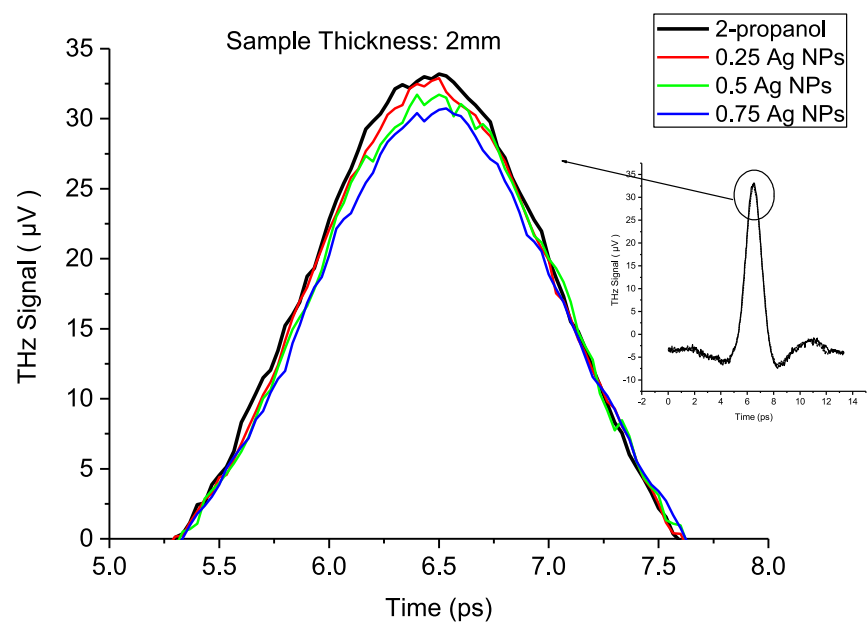

(b)

Fig. 2. (a) Refractive index and absorption data as compared with respect to 2-propanol. The Ag-NPs ratio to the host 2-propanol by volume in these samples were as follows: $0.75 \mathrm{Ag}, 0.5 \mathrm{Ag}$, and $0.25 \mathrm{Ag}$. The acquisition conditions were as follows $1 \mu \mathrm{m}$ step size for $1 \mathrm{~mm}$ of scan distance. The above absorption profiles were not corrected for the refractive index mismatch of the nanoparticle suspensions with respect to the quartz sample holder. (b) THz waveform profiles through various suspensions.

refractive index showed no discernible difference between the isopropanol host fluid and the NPs suspensions [see Fig. 2(a)]. The dispersive effects of the medium on the probe pulse can disrupt the shape of the measured $\mathrm{THz}$ pulse and consequently obstruct resolving any appreciable changes in refractive index associated with the nanoparticle additives. Since the shifts were so small that dispersive effects could negate overall analysis of the waveform only shifts around the main peak of the THz pulse was examined where the signal to noise ratio is maximized as seen in Fig. 2(b).

These selected time-domain profiles showed interesting differences such as a slight time shift and a decrease in the intensity with the increase in the NPs concentration [see Fig. 2(b)]. It is important to note that various liquids can show temperaturedependent changes in refractive index [20]. Complete datasets of measurements done here were taken within a short time frame (hours) in climate controlled laboratory conditions.

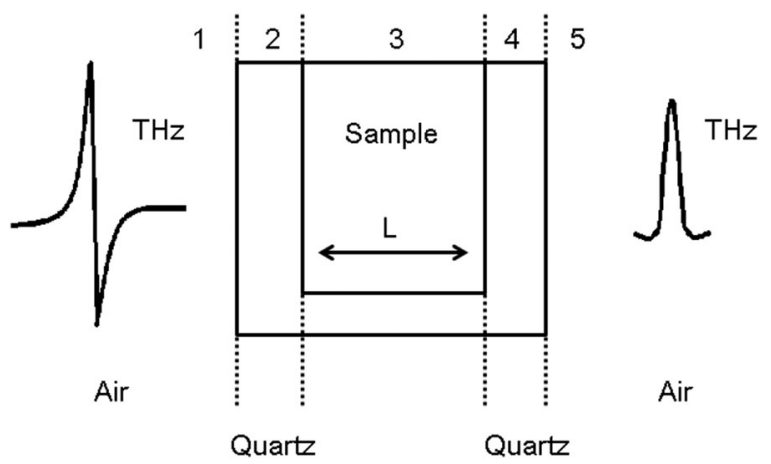

Fig. 3. THz pulse is transmitted at normal incidence over the consecutive interfaces. The 1,2,3,4, and 5 quantities refer to the mediums of air, sample holder, and the sample of thickness $L$ consecutively.

\section{TIME-DOMAIN DATA ANALYSIS}

\section{A. Sampling System and Theoretical Formulations}

To estimate the changes in the complex refractive index of the nanoparticle suspension (called NanoFluid (NF) for the purposes of this analysis from here on) with varying concentrations, the observed shifts in the peak of the $\mathrm{THz}$ pulse profile as well as changes in its amplitude were quantified by first analyzing the frequency-dependent transmission of the THz pulse through the medium and then retransforming that information into the time-domain to fit the observed waveform. Once the correct simulations of the time-domain pulse profiles were obtained, the phase and amplitude of the transmitted field was used to estimate the NF complex refractive index. Afterward, using a Maxwell-Garnett approach an assessment was made for the number of NPs in the 2-propanol fluid.

A medium whose properties are constant throughout each plane perpendicular to a fixed direction is called a stratified medium [21]. The theory of stratified media is of considerable importance in optics, in connection with multilayers. The derivation of the differential equations starting from Maxwell's equations for a time-harmonic electromagnetic wave propagated through a stratified medium and the most conventional expression of various theorems related to stratified media are given as a characteristic matrix in details in [21].

In the THz-TDS experiments, a plane wave of angular frequency $\omega$ is transmitted at normal incidence over the consecutive interfaces of air, quartz, and NF sample under a plane-wave, single pass approximation by excluding the possible internal reflections in the sample and sample windows as illustrated in Fig. 3.

In terms of Total Transmission amplitude $t_{m}$ and total phase change $\varphi_{\mathrm{m}}$, the ratio of NF (sample pulse) $\mathrm{E}_{\mathrm{NF}}$ to isopropanol solution (reference pulse) $\mathrm{E}_{\text {iso }}$ in the frequency domain is given by

$$
\frac{\mathrm{E}_{\mathrm{NF}}}{\mathrm{E}_{\mathrm{iso}}}=\mathrm{t}_{\mathrm{m}} \mathrm{e}^{-\mathrm{i} \varphi_{\mathrm{m}}} .
$$


For the geometry shown in Fig. 2, ratio of the sample pulse to reference pulse is described as [22]

$$
\frac{E_{N F}}{E_{\text {iso }}}=\frac{\left(\frac{2 n_{Q}}{n_{Q}+\widetilde{n_{N F}}}\right) e^{-i \widetilde{n_{N F}} k L}\left(\frac{2 \widetilde{n_{N F}}}{n_{Q}+\widetilde{n_{N F}}}\right)}{\left(\frac{2 n_{Q}}{n_{Q}+\widetilde{n_{\text {iso }}}}\right) e^{-i \widetilde{n_{\text {iso }}} k L}\left(\frac{2 \widetilde{n_{\text {iso }}}}{n_{Q}+\widetilde{n_{\text {iso }}}}\right)}
$$

where $n_{Q}$ is the refractive index of quartz and the complex index of refractions of isopropanol base fluid, $\widetilde{\mathrm{n}_{\mathrm{iso}}}$ and the NF solution, $\widetilde{\mathrm{n}_{\mathrm{NF}}}$ are given as

$$
\begin{aligned}
& \widetilde{\mathrm{n}_{\mathrm{NF}}}=\mathrm{n}_{\mathrm{NF}}-\mathrm{ik}_{\mathrm{NF}} \\
& \widetilde{\mathrm{n}_{\text {iso }}}=\mathrm{n}_{\text {iso }}-\mathrm{ik}_{\text {iso }} .
\end{aligned}
$$

Here, the relative Fresnel transmission coefficients are given through the interfaces 2 to 3 and 3 to 4 only. The other coefficients cancel out because they appear for both transmission through the NF sample holder and the pure 2-propanol sample holder.

In addition, the wave acquires a phase that is proportional to the complex index of the medium. Since both media are absorbing, both refractive indices are complex. By rearranging the equation in the form, $\frac{E_{N F}}{E_{\text {iso }}}=\widetilde{Z} \mathrm{e}^{\widetilde{Y}}$ where $\tilde{Y}$ contains both the argument and the loss, we get

$$
\frac{\mathrm{E}_{\mathrm{NF}}}{\mathrm{E}_{\text {iso }}}=\frac{\widetilde{\mathrm{n}_{N F}}\left(\mathrm{n}_{\mathrm{Q}}+\widetilde{\mathrm{n}_{\text {iso }}}\right)^{2}}{\widetilde{\mathrm{n}_{\text {iso }}}\left(\mathrm{n}_{\mathrm{Q}}+\widetilde{\mathrm{n}_{\mathrm{NF}}}\right)^{2}} \mathrm{e}^{-\alpha_{\mathrm{NF}} \mathrm{L}} \mathrm{e}^{\alpha_{\text {iso }} \mathrm{L}} \mathrm{e}^{-\mathrm{i}\left(\mathrm{n}_{\mathrm{NF}}-\mathrm{n}_{\text {iso }}\right) \mathrm{kL}} \text {. }
$$

Here, $\mathrm{k}=\frac{\omega}{\mathrm{c}}$ and as seen from the above equation, $\widetilde{\mathrm{Z}}$ is given as

$$
\widetilde{\mathrm{Z}}=\frac{\widetilde{\mathrm{n}_{\mathrm{NF}}}\left(\mathrm{n}_{\mathrm{Q}}+\widetilde{\mathrm{n}_{\text {iso }}}\right)^{2}}{\widetilde{\mathrm{n}_{\mathrm{iso}}}\left(\mathrm{n}_{\mathrm{Q}}+\widetilde{\mathrm{n}_{\mathrm{NF}}}\right)^{2}} .
$$

The field absorption coefficients of the base fluid and the NF are given as

$$
\begin{aligned}
\alpha_{\mathrm{NF}} & =\frac{\omega}{\mathrm{c}} \mathrm{k}_{\mathrm{NF}} \\
\alpha_{\mathrm{iso}} & =\frac{\omega}{\mathrm{c}} \mathrm{k}_{\mathrm{iso}} .
\end{aligned}
$$

Using these equations the modulus and argument of $\widetilde{\mathrm{Z}}$, becomes

$$
\begin{aligned}
|\widetilde{\mathrm{Z}}| & =\left(\frac{\mathrm{a}^{2}+\mathrm{b}^{2}}{\mathrm{c}^{2}+\mathrm{d}^{2}}\right)^{1 / 2} \\
\operatorname{Arg} \widetilde{\mathrm{Z}} & =\arctan \left(\frac{\mathrm{bc}-\mathrm{ad}}{\mathrm{ac}+\mathrm{bd}}\right)
\end{aligned}
$$

where

$$
\begin{aligned}
\mathrm{a}= & \mathrm{n}_{\mathrm{NF}} \mathrm{n}_{\mathrm{Q}}^{2}+\mathrm{n}_{\mathrm{NF}} \mathrm{n}_{\text {iso }}^{2}-\mathrm{n}_{\mathrm{NF}} \mathrm{k}_{\text {iso }}^{2}+2 \mathrm{n}_{\mathrm{Q}} \mathrm{n}_{\text {iso }} \mathrm{n}_{\mathrm{NF}} \\
& -2 \mathrm{k}_{\mathrm{NF}} \mathrm{n}_{\text {iso }} \mathrm{k}_{\text {iso }}-\mathrm{k}_{\mathrm{NF}} \mathrm{n}_{\mathrm{Q}} \mathrm{k}_{\text {iso }} \\
\mathrm{b}= & -\mathrm{i}\left(2 \mathrm{n}_{\mathrm{NF}} \mathrm{n}_{\text {iso }} \mathrm{k}_{\text {iso }}+2 \mathrm{n}_{\mathrm{Q}} \mathrm{n}_{\mathrm{NF}} \mathrm{k}_{\text {iso }}+\mathrm{k}_{\mathrm{NF}} \mathrm{n}_{\mathrm{Q}}^{2}+\mathrm{k}_{\mathrm{NF}} \mathrm{n}_{\text {iso }}^{2}\right. \\
& \left.-\mathrm{k}_{\mathrm{n}} \mathrm{k}_{\text {iso }}^{2}+2 \mathrm{k}_{\mathrm{NF}} \mathrm{n}_{\mathrm{Q}} \mathrm{n}_{\text {iso }}\right) \\
\mathrm{c}= & \mathrm{n}_{\text {iso }} \mathrm{n}_{\mathrm{Q}}^{2}+\mathrm{n}_{\text {iso }} \mathrm{n}_{\mathrm{NF}}^{2}-\mathrm{n}_{\mathrm{iso}} \mathrm{k}_{\mathrm{NF}}^{2}+2 \mathrm{n}_{\mathrm{Q}} \mathrm{n}_{\text {iso }} \mathrm{n}_{\mathrm{NF}} \\
& -2 \mathrm{k}_{\text {iso }} \mathrm{n}_{\mathrm{NF}} \mathrm{k}_{\mathrm{NF}}-2 \mathrm{k}_{\text {iso }} \mathrm{n}_{\mathrm{Q}} \mathrm{k}_{\mathrm{NF}}
\end{aligned}
$$

$$
\begin{gathered}
\mathrm{d}=-\mathrm{i}\left(2 \mathrm{n}_{\text {iso }} \mathrm{n}_{\mathrm{NF}} \mathrm{k}_{\mathrm{NF}}+2 \mathrm{n}_{\mathrm{Q}} \mathrm{k}_{\mathrm{NF}} \mathrm{n}_{\text {iso }}+\mathrm{k}_{\text {iso }} \mathrm{n}_{\mathrm{Q}}^{2}+\mathrm{k}_{\mathrm{iso}} \mathrm{n}_{\mathrm{NF}}^{2}\right. \\
\left.-\mathrm{k}_{\text {iso }} \mathrm{k}_{\mathrm{NF}}^{2}+2 \mathrm{k}_{\text {iso }} \mathrm{n}_{\mathrm{Q}} \mathrm{n}_{\mathrm{NF}}\right) .
\end{gathered}
$$

Finally, the ratio of sample pulse to reference pulse in the frequency domain is equated as

$$
\frac{\mathrm{E}_{\mathrm{NF}}}{\mathrm{E}_{\mathrm{iso}}}=|\widetilde{\mathrm{Z}}| \mathrm{e}^{\left(\alpha_{\text {iso }}-\alpha_{\mathrm{NF}}\right) \mathrm{L}} \mathrm{e}^{-\mathrm{i} \varphi_{\mathrm{m}}}
$$

where the frequency-dependent field absorption coefficient $\alpha_{\text {iso }}(\omega)$ can be calculated from the above equation by using the power transmission $\mathrm{T}$, which is computed from the FFT analysis of isopropanol with respect to quartz [23]

$$
\alpha_{\text {iso }}=-\frac{1}{2 \mathrm{~L}} \ln \left[\left(\frac{\left(\mathrm{n}_{\text {iso }}+\mathrm{n}_{\mathrm{Q}}\right)^{2}}{4 \mathrm{n}_{\mathrm{Q}} \mathrm{n}_{\mathrm{iso}}}\right)^{2} \cdot \mathrm{T}\right] \text {. }
$$

The field absorption for the NF is also corrected for reflective losses between the quartz and NF index mismatch as described in the following section.

Finally, the total transmission amplitude and total phase change are found to be

$$
\begin{aligned}
t_{\mathrm{m}} & =\left(\frac{\mathrm{a}^{2}+\mathrm{b}^{2}}{\mathrm{c}^{2}+\mathrm{d}^{2}}\right)^{1 / 2} \mathrm{e}^{\left(\alpha_{\mathrm{iso}}-\alpha_{\mathrm{NF}}\right) \mathrm{L}} \\
\varphi_{\mathrm{m}} & =\arctan \left(\frac{\mathrm{bc}-\mathrm{ad}}{\mathrm{ac}+\mathrm{bd}}\right)+\Delta \mathrm{n} \frac{\omega}{\mathrm{c}} \mathrm{L}
\end{aligned}
$$

where $\Delta \mathrm{n}=\mathrm{n}_{\mathrm{NF}}-\mathrm{n}_{\mathrm{iso}}$, and $L$ is the length of the NF or host fluid.

\section{B. Modeling the Measurements}

To obtain the extinction coefficient and refractive index of the NF sample, the above analysis was applied to the measured $\mathrm{THz}$ waveform through the isopropanol host fluid. The index of refraction $n_{\text {iso }}(\omega)$ is calculated from the experimental data and the index of refraction of quartz is $n_{\mathrm{Q}}=2.1$ [24] near $0.5 \mathrm{THz}$. The frequency-dependent phase shift $\left(\varphi_{\mathrm{m}}\right)$ is estimated by applying an appropriate phase shift to the transformed isopropanol $\mathrm{THz}$ time domain measurement until the inverse transform of this modified waveform matches the $\mathrm{THz}$ time-domain waveform through the NF. Then, once an initial estimate is made for $\varphi_{\mathrm{m}}$ using this fitting procedure, (17) and (18) are solved simultaneously to extract the refractive index and extinction coefficient of the NPs Suspension (and/or NF) where initial estimates were fed back into the equations until a satisfactory calculation for the transmission amplitude and phase is obtained. The procedure employed here is focused on determining the shifts near the peak of the THz waveform, and as such can be determined with high precision. The results of this procedure are shown for the Ag-NPs suspensions in Fig. 4. These data sets show a slight positive phase shift which can be well explained by the theory as described in the previous section.

Due to the limited frequency range of the measurement data sets, Fig. 5 shows the obtained phase shift $\varphi_{\mathrm{m}}^{\prime}$ (where the prime denotes the phase shift obtained directly from analysis of the time-domain data, given by the fitted curves) for different concentrations at three different frequencies: 0.1, 0.5, and $0.8 \mathrm{THz}$. 


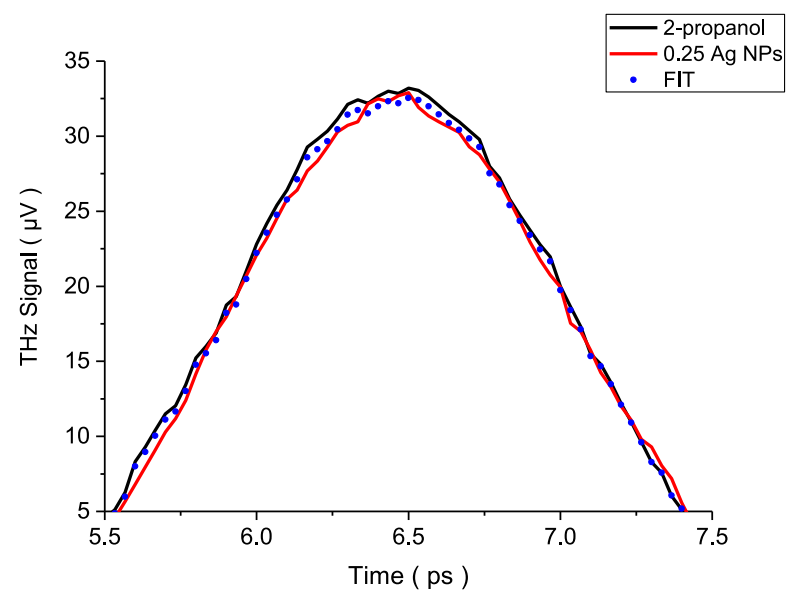

(a)

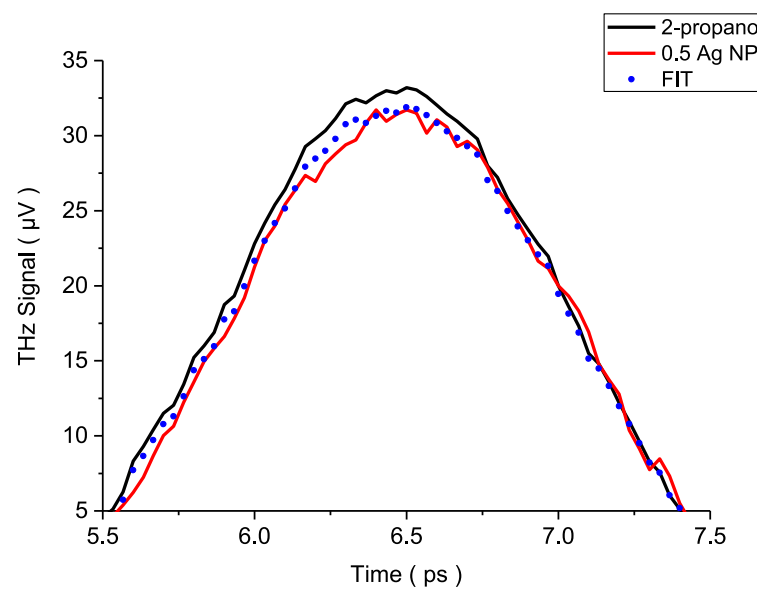

(b)

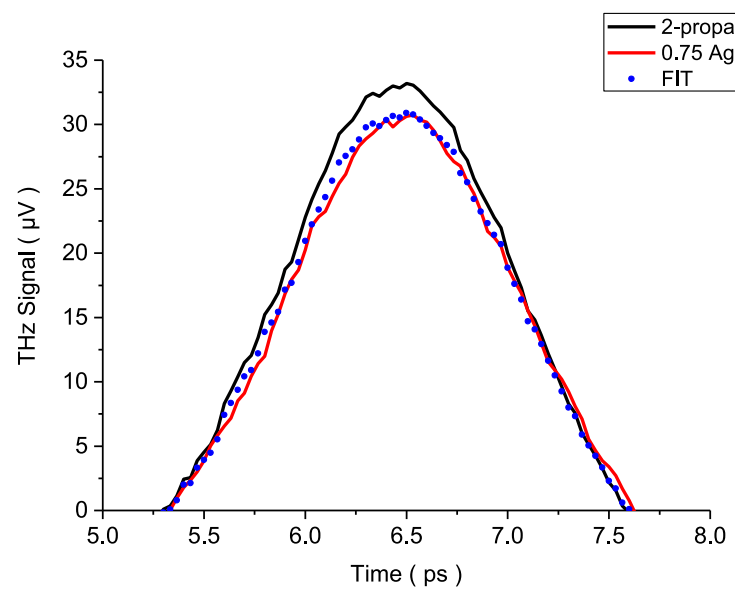

(c)

Fig. 4. THz time domain spectrum of Ag-NPs dispersions of Ag-NPs and 2-propanol reference waveform. The closed circles represent the matching fit respectively for NP volume ratio of (a) 0.25 , (b) 0.5 , and (c) 0.75 .

The phase shifts calculated and shown here assume that the refractive index of quartz is the same across the band. A frequencydependent change in the refractive index across this band will change the slope of the curves but not their overall separation.

The calculated transmission coefficient $t_{\mathrm{m}}$ at the center frequency, $0.5 \mathrm{THz}$ are tabulated in Table I.

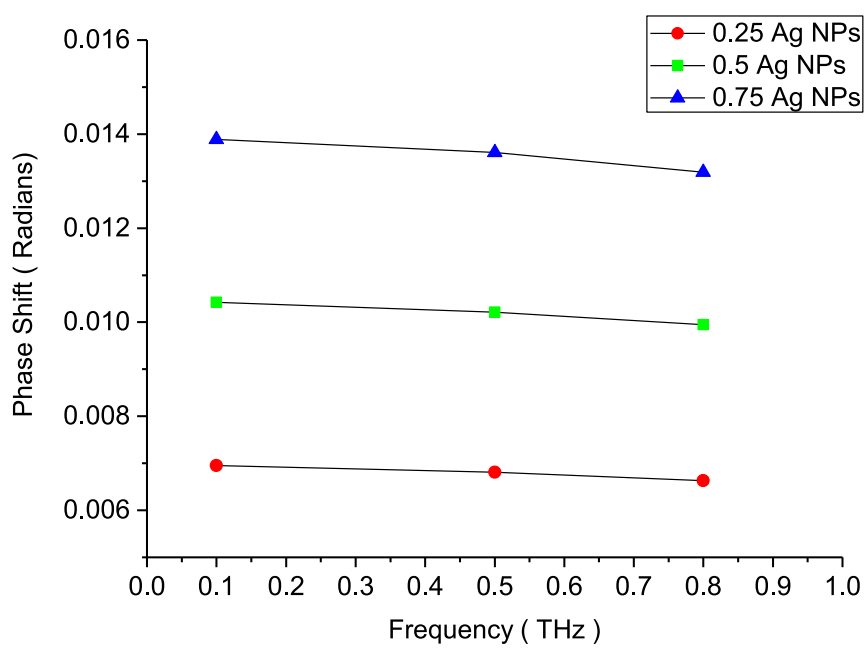

Fig. 5. Phase shift $\varphi \mathrm{m}^{\prime}$ calculated from the time domain data fits. THz waveform profiles through various suspensions.

TABLE I

Total Transmission Amplitudes Calculated From the Time Domain DATA FIT AND ABSORPTION COEFFICIENT RATIO OF AG-NPS TO ISOPROPANOL AT $0.5 \mathrm{THZ}$

\begin{tabular}{lcc}
\hline \hline Ag-NPs by Volume Ratio & $\mathrm{t}_{\mathrm{m}}$ & $\frac{\alpha_{\mathrm{NF}}}{\alpha_{\text {iso }}}$ \\
\hline 0.25 & 0.9800 & 1.0176 \\
0.5 & 0.9600 & 1.0176 \\
0.75 & 0.9300 & 1.0176 \\
\hline \hline
\end{tabular}

TABLE II

ESTIMATED INDEX OF REFRACTION OF THE GIVEN AG-NPS SUSPENSIONS AT $0.5 \mathrm{THZ}$

\begin{tabular}{lcccc}
\hline \hline THz & Refractive Index of Isopropanol & $0.25 \mathrm{Ag} \mathrm{NPs}$ & $0.5 \mathrm{Ag} \mathrm{NPs}$ & $0.75 \mathrm{Ag} \mathrm{NPs}$ \\
\hline 0.5 & 1.5923 & 1.5926 & 1.5928 & 1.5929 \\
\hline \hline
\end{tabular}

Using these values for $t_{\mathrm{m}}$ and $\varphi_{\mathrm{m}}^{\prime}$ the absorption coefficient values are calculated as given in Table I and index of refraction data for the varying concentration of Ag-NPs suspensions are given in Table II for the center of the transmission band.

\section{NPs Concentration Estimation}

The index of refraction of $\mathrm{Ag}$ was obtained from reported reflectivity measurements [25] at $0.5 \mathrm{THz}$. It is calculated to be 998 for a given reflectivity of 0.996 . Care must be taken when using bulk parameters to describe optical properties of nanoparticles. The particles in this study have sizes below the mean free path of electrons. Due to the size of the nanoparticles, a variation of the Drude model that includes possible carrier backscatter processes due to spatial confinement of the carriers is well given by the Drude-Smith model [26]. Our simulations have shown that the real part of the index of refraction does not considerably differentiate in between the Drude and the extreme case of complete backscattering for the Drude-Smith model near the frequency of $0.5 \mathrm{THz}$ considered here. These 
TABLE III

The Calculated Fill Factor at $0.5 \mathrm{THz}$

\begin{tabular}{ll}
\hline \hline $\mathrm{f}$ for $0.25 \mathrm{Ag}-\mathrm{NPs}$ & $0,00012 \pm 4.0 \%$ \\
$\mathrm{f}$ for $0.5 \mathrm{Ag}-\mathrm{NPs}$ & $0,00019 \pm 2.5 \%$ \\
$\mathrm{f}$ for $0.75 \mathrm{Ag}-\mathrm{NPs}$ & $0,00025 \pm 2.0 \%$ \\
\hline \hline
\end{tabular}

TABLE IV

AG-NPS CONCENTRATION FOR 0.25 AG-NPS, 0.5 AG-NPS, AND 0.75 Ag-NPS BY VOLUME RATIO

\begin{tabular}{lll}
\hline \hline $0.25 \mathrm{Ag}$ & $2,3 \cdot 10^{14} \pm 0,09 \cdot 10^{14}$ particle $/ \mathrm{cm}^{3}$ \\
$0.5 \mathrm{Ag}$ & $3,5 \cdot 10^{14} \pm 0,09 \cdot 10^{14}$ particle $/ \mathrm{cm}^{3}$ \\
$0.75 \mathrm{Ag}$ & $4,8 \cdot 10^{14} \pm 0,09 \cdot 10^{14}$ particle $/ \mathrm{cm}^{3}$ \\
\hline \hline
\end{tabular}

differences are more pronounced for much lower frequencies as well as for the modeled extinction coefficient.

The Maxwell-Garnett theory is one of the most widely used effective medium theories which describes the optical properties of a mixture of two materials. It is used to describe the effective dielectric of a heterogeneous medium where there are inclusions of spherical conducting particles in a dielectric host.

Refractive index and hence the dielectric constant of NF, $\epsilon_{\mathrm{NF}}$, that was found in the previous section for certain frequencies is used as the effective dielectric constant. The Ag-NPs are spherical with radius $r$ are suspended in 2-propanol solution which is the host medium with dielectric constant $\epsilon_{\text {iso }}$. For sphere sizes much smaller than the wavelength of the incident radiation, Maxwell-Garnet [27], [28] model can be employed in the explicit form as shown here

$$
\epsilon_{\mathrm{NF}}=\epsilon_{\text {iso }} \frac{\epsilon_{\mathrm{NP}}(1+2 \mathrm{f})-\epsilon_{\text {iso }}(2 \mathrm{f}-2)}{\epsilon_{\text {iso }}(2+\mathrm{f})+\epsilon_{\mathrm{NP}}(1-\mathrm{f})}
$$

where

$$
\mathrm{f}=\frac{4 \pi}{3} \mathrm{na}^{3}
$$

$f$ is the volume fraction of the embedded particles of radius a and $n$ is the particle number density.

Using the refractive index of Silver for Ag-NPs, the fill factor can be calculated from (20). The fill factor of Ag-NPs for 0.25 Ag-NPs by ratio, $0.5 \mathrm{Ag}-\mathrm{NPs}$ by ratio, and $0.75 \mathrm{Ag}-\mathrm{NPs}$ by ratio concentrations are shown in Table III.

Furthermore, using the obtained fill factors and an average $\mathrm{Ag}$ nanoparticle radius of $5 \mathrm{~nm}$ (as obtained using Zeta Potential Analyze Method) and (20) one can calculate the concentration of NPs in the host liquid, these results are tabulated in Table IV for the different concentration fluids.

The obtained concentrations agree within an order of magnitude with similar prepared samples that can be obtained commercially. Since the solutions were prepared by volumetric ratio of a high concentration NF with respect to the host fluid 2-propanol, slight variations can be expected between the prepared mixtures. The differences in between the intended to the measured volumetric concentrations can be attributed to the method of sample preparation where concentrations of the high concentration fluid was most likely nonuniform when drawn through micropipettes and added to the host liquid.

\section{CONCLUSION}

THz-TDS was used to examine the effects of Ag-NPs in a host liquid, 2-propanol. By measuring the $\mathrm{THz}$ transmission through the host fluid, which was 2-propanol, and the Ag-NPs suspension with an average diameter of $10 \mathrm{~nm}$ in 2-propanol, we were able to calculate changes in the refractive index for varying volumetric concentration of NF mixtures. The frequency dependent quantities such as power absorption coefficient and frequencydependent refractive index showed no discernible difference between the isopropanol host fluid and the silver nanoparticle suspensions when the measurements were analyzed across the entire spectrum of the $\mathrm{THz}$ waveform. Since the time-domain profiles showed notable differences such as shifts in time and in amplitude, a different approach based on shifts near the main peak of the $\mathrm{THz}$ waveform in the time-domain was used to estimate the effects of the Ag-NPs.

Using this new approach, slight changes in the complex refractive index were estimated by modeling the observed $\mathrm{THz}$ pulse peak profiles through the different NFs. The shifts were determined with high precision since the fits of the $\mathrm{THz}$ waveforms were done for the region near the peak of the pulse transmission. The iterative procedure employed to extract the phase and amplitude from (17) and (18) gave an error which was greater than the error from the uncertainty in the fit. This only works for a narrow range of frequencies near the center of the frequency spectrum where dispersive effects can be neglected. If there is significant dispersion between the NF and host liquid then the error due to the fit can exceed that obtained using the iterative procedure.

The estimation of the effective refractive index of the NF which increased for increasing concentration of nanoparticles in the 2-propanol host allowed us to estimate the nanoparticle concentration by using the accepted value of the refractive index for bulk $\mathrm{Ag}$ at $0.5 \mathrm{THz}$. By employing the MaxwellGarnett effective medium model, fill factors were estimated for different volumetric concentrations of Ag-NPs. From here, nanoparticle concentrations were obtained which agree within order of magnitude with nonaggregated Ag-NPs solutions that are commercially available [29]. Time-domain $\mathrm{THz}$ studies toward extracting material parameters typically rely on extracting these parameters across the entire useful spectrum of the pulse. With a changing signal to noise ratio across this spectrum it can be difficult to observe slight changes in parameters such as refractive index across all frequencies. The calculation of the fill factor as well as the concentration is not dependent on the accurate estimation of the refractive index of the NPs but more on the precise measurement of the refractive index of the host fluid and fluid with NPs. The fill factors calculated here begin to depend on the value of the refractive index for extremely high concentrations of NPs or for extremely precise measurements of the refractive index of the suspension. The analysis given here shows that a phase-sensitive approach applied in the time-domain to the main peak of the $\mathrm{THz}$ pulse can be useful toward extracting material parameters for highly dispersive and absorptive samples. For a medium such as metal NPs in a liquid host, this method shows that the slight shifts can be measured in the time-domain with high precision. 
Due to the noninvasive nature of the $\mathrm{THz}$ radiation, this technique can be employed in sensitive scenarios where accurate estimations of concentrations of particles are needed.

\section{REFERENCES}

[1] G. Schmid, Nanoparticles: From Theory to Application. Hoboken, NJ, USA: Wiley, 2003.

[2] G. Cao, Nanostructures and Nanomaterials, 2nd ed. London, U.K.: Imperial College Press, 2004.

[3] S. P. Jang and S. U. S. Choi, "Cooling performance of a microchannel heat sink with nanofluids," Appl. Therm. Eng., vol. 26, pp. 2457-2463, 2006.

[4] C. Koral, H. A. Vural, B. Ortaç, T. Okutucu, and H. Altan, "THz time domain spectroscopy investigation of the dynamics of metal nanoparticle suspensions in microchannels," in Proc. 8th Int. Conf. Nanosc. Nanotechnol., Jun. 25-29, 2012, Art. no. 403.

[5] J. B. Baxter and C. A. Schmuttenmaer, "Conductivity of Zno nanowires, nanoparticles, and thin films using time-resolved terahertz spectroscopy," J. Phys. Chem. B, vol. 110, pp. 25229-25239, 2006.

[6] K. Yamamoto et al., "Noninvasive inspection of C-4 explosive in mails by terahertz time-domain spectroscopy," Jpn. J. Appl. Phys., vol. 43, no. 3b, pp. L 414-L 417, 2004.

[7] M. R. Leahy-Hoppa, M. J. Fitch, X. Zheng, L. M. Hayden, and R. Osiander, "Wideband terahertz spectroscopy of explosives," Chem. Phys. Lett., vol. 434, pp. 227-230, 2007.

[8] E. Arik, H. Altan, and O. Esenturk, "Dielectric properties of diesel and gasoline by terahertz spectroscopy" J. Infrared Millim. THz Waves, vol. 35, pp. 759-769, 2014.

[9] E. Arik, C. Koral, H. Altan, and O. Esenturk, "A new method for alcohol content determination of fuel oils by terahertz spectroscopy," in Proc. 38th Int. Conf. Infrared Millim. THz Waves, 2013, Art. no. 1, doi: 10.1109/IRMMW-THz.2013.6665885.

[10] D. Mittleman, Sensing With Terahertz Radiation, vol. 85. Berlin, Germany: Springer, 2003

[11] X.-C. Zhang and J. Xu, Introduction to THz Wave Photonics. Berlin, Germany: Springer, 2009, doi: 10.1007/978-1-4419-0978-7.

[12] Y. S. Lee, Principles of Terahertz Science and Technology. Berlin, Germany: Springer, 2009.

[13] J. T. Schmuttenmaer, "Far-infrared dielectric properties of polar liquids probed by femtosecond Thz pulse spectroscopy" J. Phys. Chem., vol. 100, pp. 10373-10379, 1996.

[14] L. R. Snyder, J. J. Kirkland, and J. W. Dolan, Introduction to Modern Liquid Chromatography. Hoboken, NJ, USA: Wiley, 2010.

[15] G. C. Messina et al., "Pulsed laser ablation of a continuously-Fed wire in liquid flow for high-yield production of silver nanoparticles," Phys. Chem. Chem. Phys., vol. 15, 2013, Art. no. 3093.

[16] S. Burzhuev, A. Dana, and B. Ortaç, "Laser synthesized gold nanoparticles for high sensitive strain gauges," Sensors Actuators A, Phys., vol. 203, 2013, Art. no. 131.

[17] B. J. Kirby and E. F. Hasselbrink Jr., "Review, zeta potential of microfluidic substrates," Electrophoresis, vol. 25, pp. 187-202, 2004.

[18] J. Sturm, P. Grosse, S. Morley, and W. Theiss, "Far infrared optical properties of metal-insulator composites," Zeitschrift für Physik D Atoms, Molecules Clusters, vol. 26, pp. S 195-S 197, 1993.

[19] S. Moiseev, "Optical properties and some applications of plasmonic heterogeneous materials, metal, ceramic and polymeric composites for various uses," in Metal, Ceramic and Polymeric Composites for Various Uses J. Cuppoletti, Ed. New York City, NY, USA: Thomson Reuters Corp., 2011.

[20] L. Thrane, R. H. Jacobsen, and P. Uhd Jepsen, S. R. Keiding, "THz reflection spectroscopy of liquid water," Chem. Phys. Lett., vol. 240, pp. 330-333, 1995.

[21] M. Born and E. Wolf, Principles of Optics, 7th Ed. Cambridge, U.K.: Cambridge Univ. Press, 1999.

[22] H. Altan, "Characteristics of nanocomposites and semiconductor heterostructure wafers using Thz spectroscopy," Ph.D. dissertation, Dept. Phys., New Jersey Inst. Technol., Newark, NJ, USA, Jan. 2005.

[23] Y. Bouizem, A. Belfedal, J. D. Sib, and L. Chahed "Determination of absorption coefficients from the ratio between absorptance and transmittance measurements of weakly absorbing films," Opt. Commun., vol. 178, pp. 111-116, 2000.

[24] D. Grischkowsky, S. Keiding, M. Van Exter, and C. Fattinger, "Far-infrared time-domain spectroscopy with terahertz beams of dielectrics and semiconductors," J. Opt. Soc. Amer. B, Opt. Phys., vol. 7, no. 10, pp. 2006-2015, Oct. 1990.
[25] H. R. Giles, "Characterization of material properties at terahertz frequencies," Submillimeter-wave Technol. Lab., Univ. Massachusetts, Amherst, MA, USA, Tech. Rep. ADA462276, 1995.

[26] N. V. Smith, "Classical generalization of the Drude formula for the optica conductivity," Phys. Rev. B, Condens. Matter, vol. 64, 2001, Art. no. 155106 ,

[27] R. Ruppin, "Evaluation of extended Maxwell-Garnett theories," Opt. Commun., vol. 182, pp. 273-279, 2000.

[28] O. Levy and D. Stroud, "Maxwell-Garnett theory for mixtures of anisotropic inclusions: Application to conducting polymers," Phys. Rev. B, Condens. Matter, vol. 56, no. 13, pp. 8036-8046, 1997.

[29] Sample spec sheets of $10 \mathrm{~nm}$ silver nanoparticles (Apr. 2015) [Online]. Available: http://nanocomposix.com/

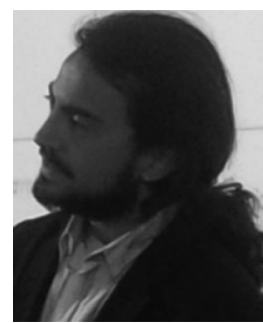

Can Koral was born in Ankara, Turkey in 1983. He received the B.S. degree in physics and M.S. degree in microtechnologies and nanotechnologies from the Middle East Technical University, Ankara, Turkey, in 2005 and 2012, respectively, and the Ph.D. degree in chemical and molecular sciences from the University of Bari Aldo Moro, Bari, Italy, in 2016.

He was a Visiting Research Scholar with the Lawrence Berkeley National Laboratory, Laser Material Interactions Group in 2015. He was involved in diverse research and development based projects as a Research Engineer with the Nanotechnology Research Center and Material Science and Nanotechnology Institution, Bilkent University, Ankara, Turkey. He is currently a Postdoctoral Research Fellow with the Department of Chemistry, University of Bari Aldo Moro, Bari, Italy. His research is focused on laser material interactions and qualitative and quantitative analyses into nanomaterials His current research interests include spectroscopy (laser induced breakdown, THz time-domain) for nanotechnology applications and nanoparticle characterization and opto-mechanical designs.

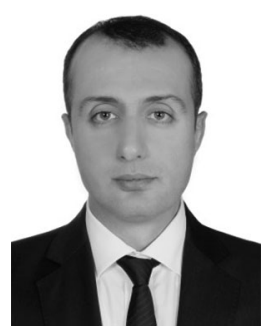

Bülend Ortaç received the B.S. degree in physics from the Karadeniz Technical University, Trabzon, Turkey, in 1997, the M.S. degree in teaching and diffusion of sciences and technology from ENS Cachan University, Paris, France, in 2000, and the Ph.D. degree in optoelectronics from Rouen University, Rouen, France, in 2004.

In March 2005, he joined the Institute of Applied Physics, Friedrich-Schiller University, Jena, Germany, as a Postdoctoral Associate. Since November 2009, he has been working as a Research Assistant Professor with the Institute of Materials Science and Nanotechnology, Bilkent University, Ankara, Turkey. He is the Founder and Principle Investigator of the Laser Research Laboratory. He has authored or coauthored more than 100 research articles in major peer-reviewed scientific journals (more than 50) and conferences (more than 90) in the field of laser physics. His research interests include the development of powerful fiber lasers in the continuous-wave regime to pulsed regime (ns, ps, and fs) and the demonstration of laser systems for real world applications.

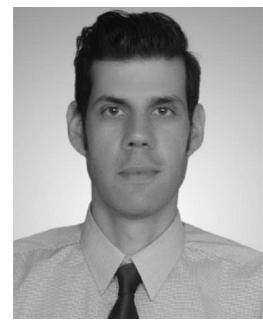

Hakan Altan received the dual B.S. degree in physics and astronomy from the State University of New York at Stony Brook, Stony Brook, NY, USA, in 1998, and the Ph.D. degree in applied physics from the New Jersey Institute of Technology, Newark, NJ, USA, in 2005.

From 2005 to 2007, he was a Member of the Senior Research Staff at the Institute for Ultrafast Spectroscopy and Lasers, City College of New York, New York, NY, USA. In 2007, he joined the Physics Department, Middle East Technical University, Ankara, Turkey, as an Assistant Professor, and in 2014, he became an Associate Professor. There he founded and continues to lead the Terahertz Research Laboratory where work is being conducted in a wide range of sub-terahertz and terahertz related fields from spectroscopy to imaging and communications.

Dr. Altan was the recipient of the Young Scientist Award from The Scientific and Technological Research Council of Turkey in 2015 and the Young Scientist Award from the Science Academy in 2016. 